\title{
A dynamic attention assessment and enhancement tool using computer graphics
}

\author{
Geeta U Navalyal ${ }^{*}$ and Rahul D Gavas
}

\author{
* Correspondence: kle.geeta@gmail. \\ com; rahulgavas@gmail.com \\ Department of Computer Science \& \\ Engg, KLE Dr. M.S.S CET, Udyambag, \\ Belgaum, India
}

\begin{abstract}
Training Programs to enhance Math Solving Skills, Memory, Visualization, etc in children are gaining popularity worldwide. Any skill is better acquired, when attention, the basic cognitive ability of the trainee is improved. This study makes an attempt to devise a technique in the form of a Brain Computer Interface (BCl) Game, to assist the trainers in monitoring and evaluating the attention levels of the trainees, at regular intervals during the training period.

The gaming environment is designed using Open Source Graphics Library (OpenGL) package and the game control is through the player's brain waves using the $\mathrm{BCl}$ technology. The players control the movement of an object from a source to a destination location on the screen by focussing their thought processes. The time taken to complete one game can be recorded. More the time taken, lesser would be the attention sustaining capacity of the player.

Thirteen subjects under different levels of the ABACUS Math Solving training program controlled the ball movement while solving math problems mentally, the time taken reduced for most of the subjects as they reached higher levels of their training course, indicating the benefit of such training programmes. The game was also played by eight non-abacus literates. The evaluation procedure was found to be very easy and fast.
\end{abstract}

Index terms: $\mathrm{ADHD} ; \mathrm{BCl} ; \mathrm{EEG}$

\section{Introduction}

Concentration or sustained attention is the basic cognitive ability of a person to perform any task or develop a skill. The timely monitoring of this ability is important when his/her performance enhancement is concerned. Though the existing methodologies to assess ones cognitive abilities in the form of mathematical, linguistic-aptitude questionnaires, face to face interaction are well established, practicing these techniques in the present day scenario seems to be time consuming and less appealing, especially in case on online training programmes. Also, many current assessment techniques stress on task completion rather than the overall process responsible for it. To improve the quality of the output, be it a task performance or a skill attainment, the entire process of it has to be monitored and evaluated [1].

Today computer games are being developed not only for entertainment of the players but in the field of health and education to improve one or more cognitive 
abilities of the users. Brain Computer Interface is one technology, which has facilitated the assessment of user behaviour through games [2]. These games can enhance problem solving abilities in children. Many "Serious" games for health and education for children are also popular [3].

The states of the brain can be recorded in a non-invasive and a flexible way through Electroencephalogram (EEG) [4]. Research has shown that EEG based applications were first developed to help disabled people to communicate with machines [5,6], then they were used in video games as game controllers [7] and then in Neurofeedback Games [8]. Children with Attention Deficit Hyperactivity Disorder (ADHD) syndrome are treated primarily with behavioural therapy than pharmacologically, as medications are bound to cause side effects [9-11].

Also training programs to help children, to focus and improve mental skills are increasingly becoming popular. Abacus is one such training program designed to develop math solving skills in children in the age groups of five to fourteen. This program is divided into many levels with increased complexity in solving skills at each level. The skills acquired at each of the levels are manually tested by specifying time limits to complete a calculation. This can be a burden on the trainer to make observations of a trainee's performance before he/she is allowed to take up the next level.

These existing techniques test the trainees solving skills, which also reflect their attention and concentration levels. So as an alternative measure, the trainees can be tested for their improvement in concentration at different stages and their progress tracked. It can also aid self evaluation to the user. This paper shows a game implementation to test the progress of the trainees with a more flexible and interesting approach. It is a simple gaming application using visualization techniques to increase enthusiasm without being aware of being tested. As the system makes use of a subject's direct attention to control a game, it works on a feedforward mechanism [12].

As inexpensive, wireless, portable and easy-to-use EEG headsets are available in the market from vendors like Neurosky and Emotiv [13,14], BCI game implementations have become very easy and assessing attention and meditation values of the users are found to be reliable [15].

Section 3 deals with the proposed game design philosophy. Game implementation details are discussed in section 4 and 5. Results of the implementation are discussed in section 6 . In section 7 and 8 , the conclusion and future work are discussed.

\section{Game design}

Any gaming application uses a graphics tool for the design of the gaming environment and user interface along with devices like keyboard, mouse or a joystick type of controllers to control the game. An EEG based game uses "thoughts" to control game. Our implementation uses OpenGL software for designing the gaming environment and an EEG device and its driver software from Neurosky.

The game design schema was done by taking into consideration the following points: The hardware used should be inexpensive and easy to maintain. It should use simple GUI so as to make a novice get accustomed to it in a short span. It was also necessary for it to use neurofeedback to give a visual representation of his attention levels and thus aid him to enhance it in a rather playful manner. 


\section{Game implementation}

The hardware includes a Lenovo Intel i5 processor based laptop and EEG device "Mindwave Mobile" from Neurosky. The implementation platform is Windows 7 and the game is developed using Visual C++ with OpenGL graphics library.

\section{Details of NeuroSky Mindwave mobile EEG}

\section{Software}

Thinkgear software provided with the EEG device is used to connect the EEG device to the computer. It allows special applications and games to be run according to the states of mind as detected by EEG headset with NeuroSky's ThinkGear sensor. It runs as a background process continuously by keeping an open socket on the computer system, thereby allowing applications to connect to it. The applications receive information from the connected ThinkGear devices. It is provided as an executable for Mac OS X and Windows platforms.

\section{Hardware}

The device is a single lobe, dry sensor electrode based, non-invasive EEG device.

ThinkGear is the technology which is embedded in every NeuroSky product. It enables connectivity with brainwaves of the wearer. It comprises of a sensor that touches the forehead to collect brain wave data centered at the frontal cortex, the contact and the reference points located on the ear pad. The data is processed using the onboard chip included on it. Both the eSense Meters (Meditation and Attention) and raw brainwaves are calculated on the ThinkGear Chip.

\section{Signal acquisition}

The MindWave Mobile Headset includes the sensor that touches the forehead, the contact and reference points located on the ear pad, and the onboard chip that processes all of the data and provides this data to software and applications in digital form through Bluetooth connectivity. Figure 1 shows the setup for the game.

The EEG electrode is placed on the user's forehead (on the frontal cortex) during the play. The headset safely measures and outputs the EEG power spectrums (alpha waves, beta waves, etc), attention, meditation and eye blink values. Attention and Meditation are indicated and reported on a meter with a relative eSense scale of 1 to 100.

This scale has a set of grouping schemata for the ranges of values and a particular state of mind is attributed to it. Values between 20-40 are reduced levels and from 1-20 they are considered strongly lowered eSense. A value lying in the range of 40-60 is considered to be neutral. Values above 60 are considered to be values higher than the normal or "slightly elevated". Values in the range of 80-100 are considered to be high levels of eSense.

Attention as an unsigned one-byte value indicates the intensity of a user's level of mental "focus" or "attention", such as that which occurs during intense concentration and directed (but stable) mental activity. Its value ranges from 0 to 100. Distractions, wandering thoughts, lack of focus or anxiety lower the Attention meter levels.

\section{Gaming strategy}

The input to this gaming application is through the attention values. The 3D ball moves from a source location to a destination location when the users focus on the task been assigned to them. Our implementation uses the eSense value 50 as a threshold value for a 


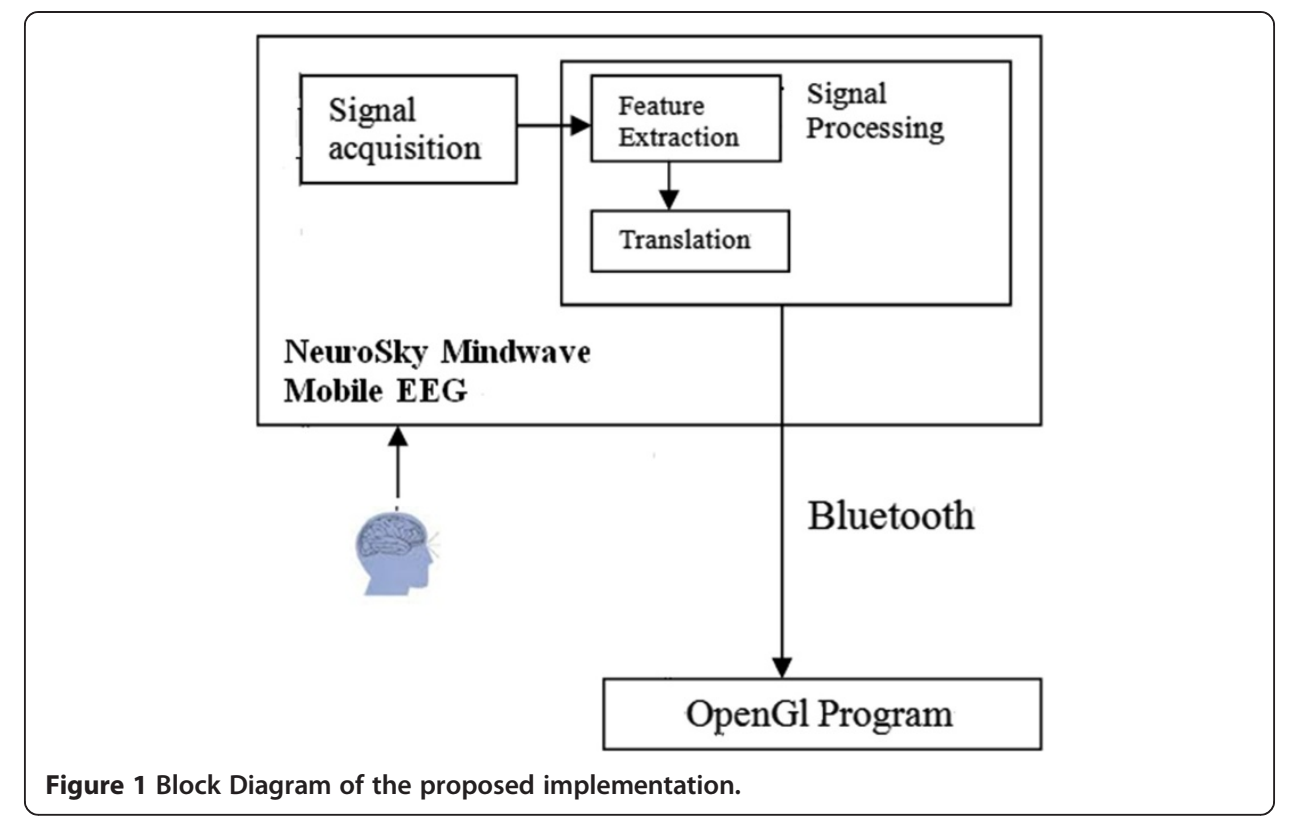

fair attention value, since it is the middlemost value lying on the eSense scale and is considered to be average balanced state of mind. When this value is crossed, the players ball moves forward towards the destination else it descends. On reaching the destination, a golden pot appears on the screen as a reward of the achievement.

\section{Methodology}

The subjects were devoid of any mental or physical disabilities and normal environmental conditions prevailed during the experimentation.

EEG data was gathered from subjects from 4 different areas and age groups.

Areas included 13 Abacus students (8-Males, 5-Females) in the age groups of 6 - 15 years, 3 under graduate students (2-males, 1-Female) in the age group of $20-22,3$ professionals (2-Males, 1-Female) in the age groups of $40-50$ and 2 senior citizens (1-Male, 1-Female) above 60 years. All of them hailed from Karnataka, India. Out of the four groups, for two groups the data was collected when they were involved in mental activities performing a task, than using the gaming tool. The subjects involved in the experimentation were either playing a game or involved in a mental activity and each of these activities induce a perceptible concentration level in the participants which the application records. The recorded data are further evaluated.

\section{Algorithm}

The following algorithm defines work flow of the proposed BCI game. The second step in the algorithm comprises of setting up proper connectivity with the EEG device and the system, initializing OpenGL features like lighting, shading and material properties and drawing the required graphics objects on the OpenGL window.

Input: Attention eSense values.

Output: Attention assessment using computer graphics. 
1. Start.

2. Set up the environment for the game.

3. Read the input and start the timer.

4. If (input value $>$ threshold).

Move the graphics object forward.

Else.

Move it backward.

5. When the graphics object reaches the destination, stop timer.

6. Stop.

Figure 2 shows the snapshot of the BCI game developed using OpenGL.

\section{Results}

Figure 3 shows the graph of abacus training levels on the $\mathrm{X}$-axis and the time taken in seconds to accomplish the BCI task assigned to them on the Y-axis.

The experimentation was carried out on students from various levels of abacus training. The students in the lower abacus training levels deal with simple addition and subtraction problems. Hence they performed better.

The students in the higher abacus training levels had to perform floating point calculations and were given multiple operations, so switching between multiple tasks hindered their attention. But the students, who had practised for more time in every level, did not panic and could perform calculations at higher attention levels.

Nervousness curbs attention values whereas confidence, self-esteem enhances it.

Figure 4 shows the graph of the age of non-abacus practitioners in years on the $\mathrm{X}$-axis and the time taken in seconds to play the game on the $\mathrm{Y}$-axis.

Attention can be divided into classes of ages. Too young (below 10 years) and too old ones (above 70 years) lack attention whereas teenagers are consistent in this regard.

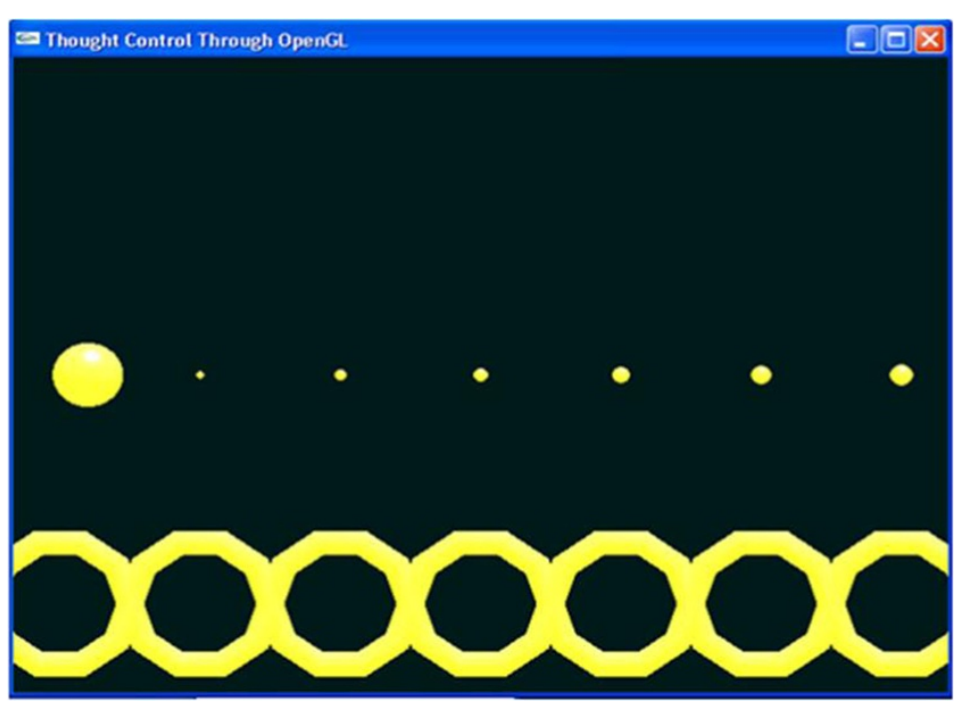

Figure 2 Snapshot of the $\mathrm{BCl}$ game. 


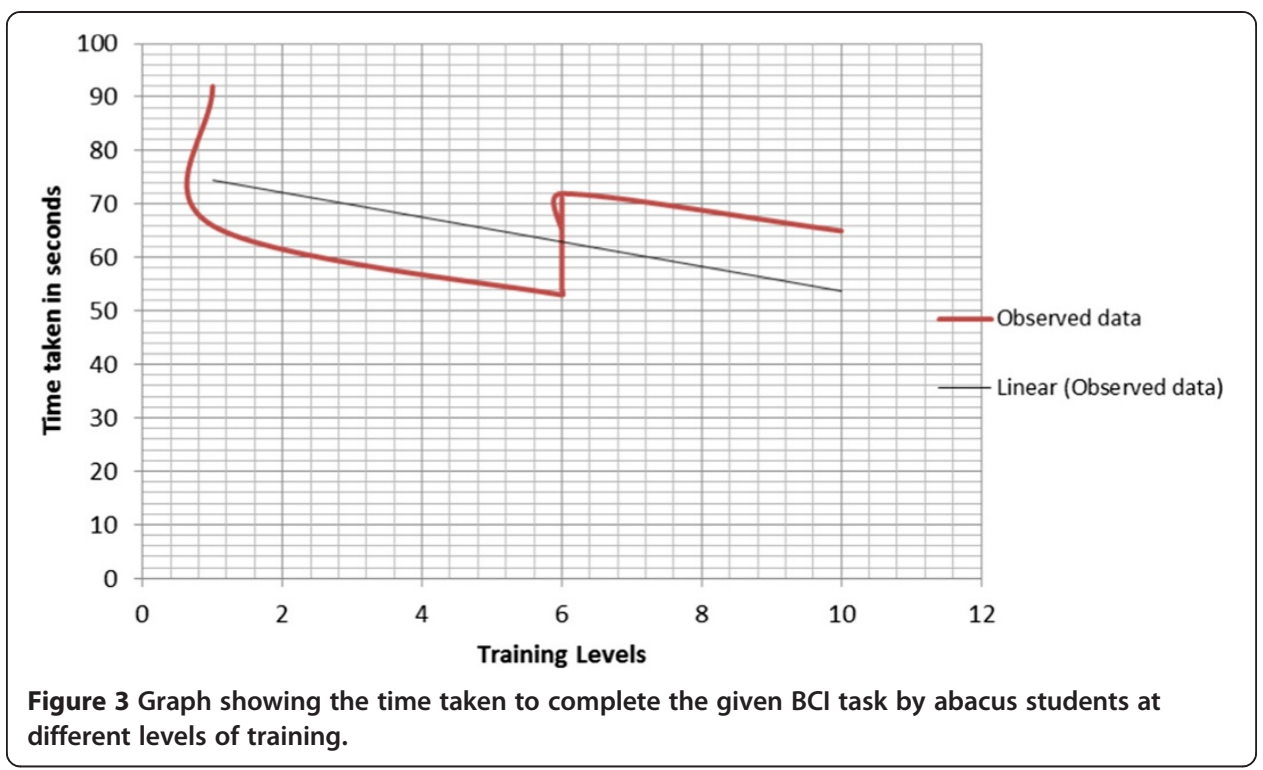

Further it considerably reduces with age. The subjects selected were not trained with special mental skills.

The linear trendline is a best-fit straight line for the data sets obtained which follows the equation given below to calculate the least squares fit for a line:

$\mathrm{y}=\mathrm{mx}+\mathrm{c}$, where $\mathrm{m}$ is the slope and $\mathrm{c}$ is the intercept.

The application was tested on an assortment of subjects from mind training institutes like abacus institute. Surprisingly, these subjects could navigate the sphere with greater ease in contrast with the normal subjects.

\section{Conclusion}

As a summary, the paper draws sharp inferences of the mental activities with conjunction to the factors like age, mental training, etc. The visual game aids as a tool in this regard. The graphical analysis reveals the correlation that exists between the attention values and their enhancement for subjects undergoing mental training skills like abacus and non-abacus subjects. It also reveals the

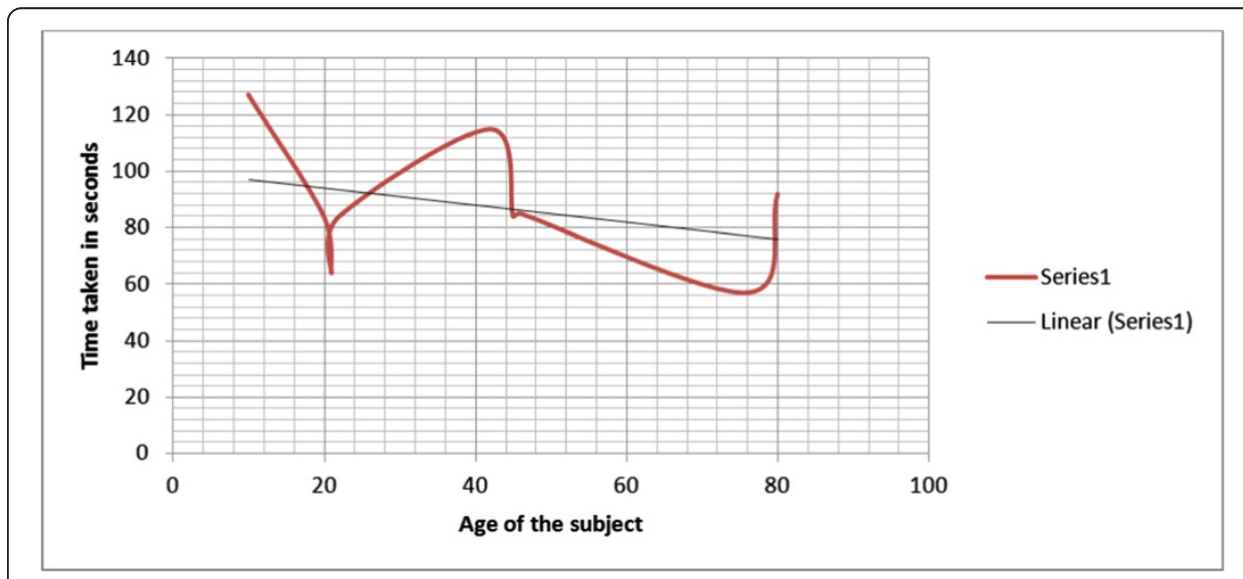

Figure 4 Graph showing the time taken by non-abacus practitioners to play the $\mathrm{BCl}$ game. 
benefits of such training programmes. The application has the ability of providing feedback to children on their performance and monitoring their progress over time.

\section{Future work}

Visual modelling features of OpenGL can be added to distract a user during the game, which through control of thoughts could be overcome and could aid children with ADHD disorder to improve concentration. Playing such games on regular basis could enhance one's attention and focussing capabilities considerably.

\section{Authors' contributions}

Both the authors, GU and RG, have equally contributed in implementation of the proposed system, data acquisition and analysis, and documentation. All authors read and approved the final manuscript.

\section{Author's information}

Prof Geeta N. has graduated in Electronics and Communication Engineering from GIT, Belgaum and completed Post-graduation in Computer Networking from VTU Belgaum. Currently she is working as Assoc. Prof in Computer Science and Engineering at KLE Dr. M.S.S College of Engineering and Technology, Belgaum, Karnataka. Her areas of interest include cognitive computing, brain-computer interface, computer networks and parallel processing. Mr. Rahul Dasharath Gavas is pursuing his Bachelor of Engineering in Computer Science and Engineering from KLE Dr. M.S.S College of Engineering and Technology, Belgaum, India. His areas of interest include cognitive computing brain-computer interface and unpredictability theory.

\section{Acknowledgements}

The authors are immensely grateful to the valuable guidance provided by Dr. Nandini Sidnal, HOD, Department of Computer Science \& Engg, KLE Dr. M.S.S CET, Udyambag, Belgaum.

Received: 12 November 2013 Accepted: 20 May 2014

Published online: 26 July 2014

\section{References}

1. Ferris GR, Munyon TP, Basik K, Buckley MR (2008) The Performance Evaluation Context: Social, Emotional, Cognitive, Political, and Relationship Components, Human Resource Management Review 18. Elsevier, pp 146-163, doi:10.1016/j.hrmr.2008.07.006

2. Payam Aghaei P, Tauseef G, Omar AZ, Gaetano G, Calvo RA (2008) Brain-Computer Interface: Next Generation Thought Controlled Distributed Video Game Development Platform. IEEE Symposium on Computational Intelligence and Games (CIG'08). doi:10.1109/CIG.2008.5035647

3. Wang Q, Sourina O, Nguyen MK (2010) EEG-based "Serious" Games Design for Medical Applications. Cyberworlds, International Conference, Singapore, IEEE Computer Society. doi:10.1109/CW.2010.56

4. Nunez PL, Srinivasan R (2006) Electric fields of the brain: the neurophysics of EEG. Oxford University Press, Inc New York

5. Rebsamen B, Burdet E, Guan C, Zhang H, Teo CL, Zeng Q, Ang M, Laugier C (2006) A brain-controlled wheelchair based on P300 and path guidance. Biomedical Robotics and Biomechatronics. The First IEEE/RAS-EMBS International Conference. pp 1101-1106, doi:10.1109/BIOROB.2006.1639239

6. Rebsamen B, Teo CL, Zeng Q, Ang VMH, Burdet E, Guan C, Zhang H, Laugier C (2006) Controlling a wheelchair indoors using thought. IEEE Intelligent Systems 22:18-24, 2007. doi:10.1109/MIS.2007.26

7. Lécuyer A, Lotte F, Reilly RB, Leeb R, Hirose M, Slater M (2008) Brain-computer interfaces, virtual reality, and videogames. Computer 41:66-72

8. Hammond DC (2006) What is neurofeedback? Journal of Neurotherapy 10:25-36, 2006. doi:10.1300/J184v10n04_04

9. Lim CG, Lee TS, Guan C, Sheng Fung DS, Cheung YB, Teng SS, Zhang H, Krishnan KR (2010) Effectiveness of a brain-computer interface based programme for the treatment of ADHD: a pilot study. Pscyopharmacological Bulletin 43(1):73-82

10. Gevensleben H, Birgit H, Björn A, Schlamp D, Kratz O, Studer P, Wangler S, Rothenberger A, Moll GH, Heinrich H (2009) Distinct EEG effects related to neurofeedback training in children with ADHD: A randomized controlled trial. International Journal of Psychophysiology 74:149-157, doi:10.1016/j.ijpsycho.2009.08.005

11. Cowan JD, Markham L (1994) EEG biofeedback for the attention problems of Autism - A case study. Biofeedback and Self-Regulation 19:287-287

12. Lopetegui E, Zapirain BG, Mendez A (2011) Tennis Computer Game With Brain Control Using EEG Signals. Computer Games (CGAMES), 2011 16th International Conference. doi:10.1109/CGAMES.2011.6000344

13. Neurosky Mindwave Mobile. Available at: http://www.Neurosky.com

14. Emotive EEG System. Available at: http://www.emotiv.com

15. Crowley K, Aidan S, Pitt I, Murphy D (2010) Evaluating a brain-computer interface to categorise human emotional response. ICALT doi:10.1109/ICALT.2010.81 